\title{
Real-Life Clinical Data of Cabozantinib for Unresectable Hepatocellular Carcinoma
}

Francesco Tovoli ${ }^{a}$ Vincenzo Dadduzio $^{b}$ Stefania De Lorenzo ${ }^{c}$ Lorenza Rimassa $^{\text {d, e }}$ Gianluca Masi ${ }^{\mathrm{f}}$ Massimo lavaroneg Fabio Marra $^{\text {h }}$ Ingrid Garajova ${ }^{i}$ Maria Pia Brizzi ${ }^{j}$ Bruno Daniele ${ }^{k}$ Franco Trevisanil, m Carlo Messina $^{\mathrm{n}}$ Francesco Di Clemente $^{\circ}$ Sara Pini $^{p}$ Giuseppe Cabibbo $^{q}$ Alessandro Granito $^{a}, \mathrm{~m}$ Mario Domenico Rizzato ${ }^{b}, \mathrm{r}$ Vittorina Zagonel ${ }^{\mathrm{b}}$ Giovanni Brandi $^{c}$ Tiziana Pressiani $^{\mathrm{e}}$ Piera Federico $^{k}$ Caterina Vivaldi $^{f}$ Irene Bergnag ${ }^{\text {Claudia Campani }}{ }^{\text {h }}$ Fabio Piscaglia ${ }^{a, m}$

\begin{abstract}
aDivision of Internal Medicine, Hepatobiliary and Immunoallergic Diseases, IRCCS Azienda Ospedaliero-Universitaria di Bologna, Bologna, Italy; ${ }^{b}$ Medical Oncology Unit 1, Istituto Oncologico Veneto, IRCCS, Padova, Italy; ${ }^{c}$ Medical Oncology, IRCCS Azienda Ospedaliero-Universitaria di Bologna, Bologna, Italy; ${ }^{\mathrm{d} D e p a r t m e n t}$ of Biomedical Sciences, Humanitas University, Pieve Emanuele, Milan, Italy; ${ }^{\mathrm{e} M e d i c a l ~ O n c o l o g y}$ and Hematology Unit, Humanitas Cancer Center, IRCCS Humanitas Research Hospital, Rozzano, Milan, Italy; ${ }^{f}$ Department of Medical Oncology, Pisa University Hospital, Pisa, Italy; ' ${ }^{D}$ ivision of Gastroenterology and Hepatology, Foundation IRCCS Ca' Granda Ospedale

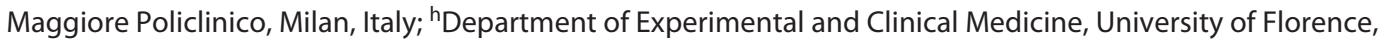
Florence, Italy; 'Medical Oncology Unit, University Hospital of Parma, Parma, Italy; ${ }^{j}$ Medical Oncology Unit, A.O.U. S. Luigi Gonzaga, Orbassano, Italy; kU.O.C. Oncologia, Ospedale del Mare, Napoli, Italy; 'Semeiotica Medica, IRCCS Azienda Ospedaliero-Universitaria di Bologna, Bologna, Italy; ${ }^{m}$ Department of Medical and Surgical Sciences,

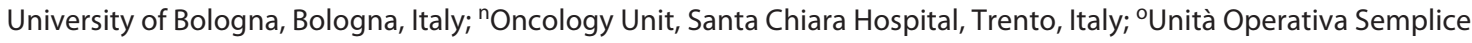
Dipartimentale (UOSD) “Oncologia medica” del Valdarno, Montevarchi, Italy; PDivision of Oncology, AUSL Romagna, Rimini, Italy; ${ }^{9}$ Section of Gastroenterology \& Hepatology, Department of Health Promotion Sciences Maternal and Infant Care, Internal Medicine and Medical Specialties, PROMISE, University of Palermo, Palermo, Italy; 'Department of Surgery, Oncology and Gastroenterology, University of Padua, Padua, Italy
\end{abstract}

\section{Keywords}

Hepatocellular carcinoma - Cabozantinib · Sorafenib .

Tyrosine kinase inhibitors · Outcome

\begin{abstract}
Introduction: Cabozantinib has been approved by the European Medicine Agency (EMA) for hepatocellular carcinoma
\end{abstract}

(HCC) previously treated with sorafenib. Cabozantinib is also being tested in combination with immune checkpoint inhibitors in the frontline setting. Real-life clinical data of cabozantinib for HCC are still lacking. Moreover, the prognostic factors for HCC treated with cabozantinib have not been investigated. Methods: We evaluated clinical data and outcome of HCC patients who received cabozantinib in the legal context of named patient use in Italy. Results: Ninety-six pa- karger@karger.com www.karger.com/lic

Karger $\stackrel{\text { ' }}{5}$

BOPEN ACCESS
(C) 2021 The Author(s)

Published by S. Karger AG, Basel

This is an Open Access article licensed under the Creative Commons Attribution-NonCommercial-4.0 International License (CC BY-NC) (http://www.karger.com/Services/OpenAccessLicense), applicable to the online version of the article only. Usage and distribution for commercial purposes requires written permission.
Correspondence to:

Francesco Tovoli, francesco.tovoli2@unibo.it 
tients from 15 centres received cabozantinib. All patients had preserved liver function (Child-Pugh A), mostly with an advanced HCC (77.1\%) in a third-line setting (75.0\%). The prevalence of performance status (PS) $>0$, macrovascular invasion (MVI), extrahepatic spread, and alpha-fetoprotein (AFP) $>400 \mathrm{ng} / \mathrm{mL}$ was $50.0,30.2,67.7$, and $44.8 \%$, respectively. Median overall survival (OS) and progression-free survival were 12.1 (95\% confidence interval 9.4-14.8) and 5.1 (3.3-6.9) months, respectively. Most common treatment-related adverse events (AEs) were fatigue (67.7\%), diarrhoea (54.2\%), anorexia (45.8\%), HFSR (43.8\%), weight loss (24.0\%), and hypertension (24.0\%). Most common treatment-related Grade 3-4 AEs were fatigue (6.3\%), HFSR (6.3\%), and increased aminotransferases (6.3\%). MVI, ECOG-PS > 0, and AFP $>400 \mathrm{ng} / \mathrm{mL}$ predicted a worse OS. Discontinuation for intolerance and no new extrahepatic lesions at the progression were associated with better outcomes. Conclusions: In a real-life Western scenario (mostly in a third-line setting), cabozantinib efficacy and safety data were comparable with those reported in its registration trial. Data regarding the prognostic factors might help in patient selection and design of clinical trials.

(c) 2021 The Author(s)

Published by S. Karger AG, Basel

\section{Introduction}

Cabozantinib is a multi-target tyrosine kinase inhibitor (TKI) inhibiting multiple pathways involved in tumour growth and proliferation, including MET, VEGFR-2/KDR, and RET [1]. Cabozantinib has been tested as a possible therapeutic agent for hepatocellular carcinoma (HCC) in the phase 3 CELESTIAL trial [2]. This study enrolled $>700$ patients with a preserved liver function who had progressed or were intolerant to sorafenib. Patients who had received a single additional line of therapy were also included [2]. Patients were randomized to cabozantinib or placebo in 2:1 ratio [2]. Cabozantinib was superior to placebo, with a median overall survival (OS) of 10.2 versus 8.0 months (hazard ratio [HR]: $0.76,95 \%$ confidence interval [CI] $0.63-0.92, p=0.005)$ and a median progression-free survival (PFS) of 5.2 versus 1.9 months (HR 0.44, 95\% CI 0.36-0.52, p<0.001) [2]. Based on these results, the Food and Drugs Administration and the European Medicines Agency (EMA) extended the indications of cabozantinib to HCC in sorafenib-experienced patients.

The whole therapeutic scenario for HCC is expected to change dramatically in the very near future $[3,4]$, especially as the combination of the immune checkpoint in- hibitor (ICI) atezolizumab and the anti-vascular endothelial growth factor agent bevacizumab has recently demonstrated superiority compared to sorafenib (the standard of care for the last 12 years) in the randomized phase 3 IMbrave 150 trial [5].

However, the interest towards cabozantinib remains high as it is being tested in combination with atezolizum$\mathrm{ab}$ in the frontline setting versus sorafenib in the COSMIC-312 study [6], another phase 3 randomized clinical trial which had been designed in parallel with the IMbrave150 study. Moreover, the encouraging results of a cohort of the CheckMate 040 phase 1/2 study testing the combination nivolumab + cabozantinib versus nivolum$a b+$ ipilimumab + cabozantinib [7] have been released as a communication at a recent medical meeting. Regardless of the ongoing trials, cabozantinib can be already considered a viable third-line treatment following atezolizumab-bevacizumab and sorafenib (the latter being the only per-label second-line drug following atezolizumab-bevacizumab).

In this complex and rapidly evolving scenario, clinical data about cabozantinib are needed to understand whether the safety and efficacy data of its clinical trial can also be applied to the real-life clinical setting. While these data are broadly available for other TKI such as sorafenib (which is being prescribed since 2007) [8-10], there is a lack of such information for cabozantinib. Since the CELESTIAL trial was stopped at the second interim analysis, further safety and efficacy data would be desirable. Also, new information is of critical importance given the continuous therapeutic evolutions.

The EMA granted cabozantinib an approval for the treatment of HCC in September 2018. According to the Italian regulatory aspects, further approval from the Italian Drugs Agency (AIFA) is required before a new drug can be freely prescribed in everyday clinical practice. However, manufacturing companies can provide EMAapproved drugs, free of charge, for patients without therapeutic alternatives, after an individual request by physicians, in the legal context of named patient use (NPU). Since AIFA approved cabozantinib in September 2020, a sizeable number of patients received cabozantinib in a NPU setting in the preceding 2 years. This possibility has still to become available for ramucirumab, another second-line drug which showed efficacy in patients with high-alpha-fetoprotein (AFP). This investigator-driven study aims to describe the clinical characteristics and outcomes of HCC patients who received cabozantinib in real-life clinical practice, thanks to the legal context of NPU. 


\section{Methods}

Clinical Setting and Regulatory Aspects

The local Ethics Committee (EC) approved each NPU request of the proposing physician. The EC evaluated extensive documentation including a clinical report, a protocol for the follow-up of the patient, information notes and consents (participation to NPU, privacy). The protocol broadly adopted the EMA recommendations for patients receiving cabozantinib in terms of frequency and modalities of follow-up.

For this study, we subsequently analysed the clinical characteristic and outcome of patients who received cabozantinib in the NPU context. The variables subsequently utilized as baseline data were prospectively collected for all the patients, as they were required to verify the eligibility. This factor abolished the risk of missing information, which could instead be faced in retrospective studies. Also, events occurring during the follow-up, in particular adverse events (AEs), were prospectively collected and notified to the pharmacovigilance service of the manufacturing company as per the current legal requirements.

\section{Baseline Evaluation}

The following baseline information were required either by the manufacturing company and/or by the EC and were prospectively collected for each patient: age, sex, liver function tests, tumour staging according to the Barcelona Clinic for Liver Cancer (BCLC), performance status (PS) according to the Eastern Cooperative Oncology Group (ECOG-PS), alpha-fetoprotein, previous treatments for HCC.

\section{Cabozantinib Prescription}

Cabozantinib was prescribed at the approved initial dose of $60 \mathrm{mg}$ once daily. After obtaining the patient's consent, the prescribing physician provided the patient with the first bottle of cabozantinib (containing 30 tablets) and scheduled the follow-up evaluations.

At the time of the first prescription, all patients were provided with a daily record book and educated about cabozantinib-related AEs to allow an early recognition, notification, and treatment. $\mathrm{Pa}$ tients were also advised to contact their respective centres in case of new symptoms.

Dose modifications in response to the AEs (including dose reductions to 40 and $20 \mathrm{mg}$ once daily and dose stops) were performed according to the manufacturer's recommendations.

\section{Follow-Up Schedule}

The follow-up evaluations were performed according to the protocol approved the EC, which reflected the EMA recommendations of a stricter follow-up during the first months of administration. All centres were expert in the administration of systemic drugs for HCC and adopted the same follow-up modalities.

Briefly, follow-up visits were scheduled every 2 weeks for the first 8 weeks of treatment, then every 4 weeks. At each visit, patients also underwent the laboratory tests needed to exclude relevant toxicities. The first radiological evaluation of response was scheduled 8-10 weeks after the first dose of cabozantinib, with the subsequent radiological assessments scheduled every 12 weeks.

\section{Clinical Evaluation}

New symptoms arising after the treatment start, as well as their timing, were thoroughly recorded. The medical records included both a brief description of each $\mathrm{AE}$ and its coding according to the
Common Terminology Criteria for Adverse Events version 5.0. Dose modifications in response to the AEs (including dose reductions and dose interruptions) were performed according to the manufacturer's recommendations.

\section{Imaging Evaluation}

Computed tomography of the thorax, abdomen, and pelvis with iodinated contrast medium was the preferred imaging technique. For patients with contraindications to the iodinated contrast medium, magnetic resonance imaging of the abdomen paired with a high-resolution chest computed tomography was performed. Radiological evaluation of response during follow-up was performed according to the Response Evaluation Criteria in Solid Tumours v1.1 [11]. The pattern of radiological progression was categorized, as previously described by Reig et al. [12]. Briefly, 3 different categories were used: patients with baseline intermediatestage disease who progressed in size or number of intrahepatic lesions, but did not develop a new extrahepatic lesion or macrovascular invasion (MVI) (BCLCp-B), patients with baseline advancedstage disease who did not develop any new extrahepatic lesions or MVI (BCLCp-C1), and patients who developed new extrahepatic lesions and/or MVI, regardless of their initial stage (BCLCp-C2) [12].

\section{Permanent Discontinuation of Cabozantinib}

The prescription of cabozantinib was renovated every 4 weeks after a careful assessment of the clinical benefit. Cabozantinib was continued until: (1) radiological and clinical progression; (2) unacceptable AEs, or (3) deterioration of liver function. Categorization into these classes was performed according to the same criteria previously proposed by Iavarone and colleagues [13].

\section{Survival Evaluations}

OS was measured from the date of starting cabozantinib until the date of death. PFS was defined as the interval between the first dose of cabozantinib and progression or death, whichever occurred first. Time to progression was measured from the start of cabozantinib to progression. Post-cabozantinib survival (PCS) was calculated from the last day of treatment to death [13]. Post-progression survival (PPS) was measured from the first evidence of progression to death [12]. A cumulative survival from the first day of the frontline systemic drug and death was considered as a variable of interest to assess the potentiality of sequential treatments (in particular sorafenib-regorafenib-cabozantinib and sorafenibcabozantinib) [14].

\section{Statistics}

Continuous variables are expressed as median and interquartile range (IQR). Categorical variables are expressed as frequencies. Group comparisons were performed with the Mann-Whitney U test. Categorical variables were evaluated using 2-tailed Fisher's test. Survival curves were estimated using the product-limit method of Kaplan-Meier. The role of stratification factors was analysed with log-rank tests. To define the predictors of OS, we used a timedependent covariates survival approach, including statistically significant clinical variables $(p<0.05)$ from the univariate Cox analysis. Statistical analyses were performed using SPSS version 23.0 (SPSS Inc., Chicago, IL, USA). 


\section{Results}

\section{Study Population}

This study included 96 patients from 15 Italian centres (Table 1). Most patients were cirrhotic (94.8\%). The aetiology of the underlying liver disease was as follows: nonviral causes $40.6 \%$, hepatitis $C$ virus infection $32.3 \%$, and hepatitis B virus infection 27.1\%. Cabozantinib was prescribed as a third-line drug in most of the patients (79.1\%). The remaining patients received cabozantinib in the second-line setting as they were not eligible for regorafenib according to the AIFA refunding policies (liver transplant recipients $n=10$; intolerant to sorafenib $n=9$; regorafenib still not approved $n=2$ ). A total of 7 patients had received ICIs previous to cabozantinib (4 in the frontline and 3 in the second-line setting).

\section{Baseline Tumour Staging and Liver Function \\ Parameters}

All patients had preserved liver function (Child-Pugh A). Most patients had an advanced stage HCC (78.1\%) due to the presence of MVI (MVI, 30.2\%) and/or extrahepatic spread (EHS, 68.8\%). The rates of ECOG-PS $>0$ and AFP $>400 \mathrm{ng} / \mathrm{mL}$ were 50.0 and $42.7 \%$, respectively (Table 1 ).

\section{Efficacy Data}

Patients were observed for a median follow-up of 8.6 months (IQR 5.0-12.9). The median OS was 12.1 months (95\% CI 9.4-14.8) (Fig. 1). The median PFS was 5.1 months (95\% CI 3.3-6.9) and (Fig. 2). The median time to progression was 5.2 months (95\% CI 3.1-7.3). Five patients (5.2\%) died before the first imaging follow-up, in a clinical setting of early progression. The disease control rate was 63.5\%, with 4 partial responses (4.2\%) (Fig. 3). Amongst the 74 patients who experienced a radiological progression, 8 were classified as BCLCp-B, 26 as BCLCp$\mathrm{C} 1$, and 40 as BCLCp-C2.

The cumulative survival calculated from the start of a frontline systemic treatment to death was 35.4 months (95\% CI 26.9-43.9). The most frequent sequential treatments were sorafenib-regorafenib-cabozantinib $(n=51)$ and sorafenib-cabozantinib $(n=20)$ (Fig. 4). The median cumulative survival of the sequence sorafenib-regorafenibcabozantinib was 35.4 months (95\% CI 27.9-43.0), while the median cumulative survival of the sequence sorafenibcabozantinib was 20.8 months (95\% CI 14.1-27.5).

\section{Safety Data}

Overall, 91 patients (94.8\%) experienced at least 1 treatment-related AE (trAE) (Table 2). The most common
Table 1. General characteristics of the study population $(n=96)$

\begin{tabular}{|c|c|}
\hline Variable & \\
\hline Age, years & $68(59-73)$ \\
\hline Male sex & $79(82.3)$ \\
\hline \multicolumn{2}{|l|}{ Underlying liver disease } \\
\hline HBV & $26(27.1)$ \\
\hline $\mathrm{HCV}$ & $31(32.3)$ \\
\hline Non-viral & $39(40.6)$ \\
\hline \multicolumn{2}{|l|}{ Child-Pugh class } \\
\hline A5 & $53(55.2)$ \\
\hline A6 & $43(44.8)$ \\
\hline \multicolumn{2}{|l|}{ ALBI grade } \\
\hline ALBI-1 & $29(31.2)$ \\
\hline ALBI-2 & $62(64.6)$ \\
\hline ALBI-3 & $5(5.2)$ \\
\hline ECOG-PS $>0$ & $48(50.0)$ \\
\hline MVI & $29(30.2)$ \\
\hline Extrahepatic spread & $65(67.7)$ \\
\hline $\mathrm{AFP}>400 \mathrm{ng} / \mathrm{mL}$ & $43(44.8)$ \\
\hline \multicolumn{2}{|l|}{ BCLC stage } \\
\hline Intermediate & $22(22.9)$ \\
\hline Advanced & $74(77.1)$ \\
\hline \multicolumn{2}{|l|}{ Previous therapies } \\
\hline OLT & $15(15.6)$ \\
\hline Liver resection & $36(37.5)$ \\
\hline Percutaneous treatments & $39(40.6)$ \\
\hline TACE & $42(43.8)$ \\
\hline SIRT & $13(13.5)$ \\
\hline SBRT & $7(7.3)$ \\
\hline \multicolumn{2}{|l|}{ Frontline systemic treatment } \\
\hline Sorafenib & $90(93.8)$ \\
\hline Other* & $6(6.2)$ \\
\hline \multicolumn{2}{|l|}{ Second-line systemic treatment } \\
\hline None (cabozantinib) & $21(21.9)$ \\
\hline Regorafenib & $51(53.1)$ \\
\hline Sorafenib & $6(6.2)$ \\
\hline Other** & $18(18.8)$ \\
\hline
\end{tabular}

Categorical variables are reported as frequencies (\%). Continuous variable are expressed as median (IQR). HBV, hepatitis B virus; $\mathrm{HCV}$, hepatitis $\mathrm{C}$ virus; OLT, orthotopic liver transplant; TACE, transarterial chemoembolization; SIRT, selective internal radiotherapy; SBRT, stereotactic body radiation therapy; AFP, alpha-fetoprotein; BCLC, Barcelona clinic for liver cancer; MVI, macrovascular invasion; IQR, interquartile range. * Anti-programmed death protein-1 agents $(n=3)$, metronomic capecitabine $(n=2)$, and lenvatinib $(n=1)$. ${ }^{* *}$ Metronomic capecitabine $(n=$ $9)$, anti-programmed death protein-1 agents $(n=4)$; milciclib $(n=$ $1)$, conventional chemotherapy $(n=2)$, mTOR inhibitors $(n=1)$, and lenvatinib $(n=1)$.

trAEs occurring in at least $20 \%$ of the patients were fatigue (67.7\%), diarrhoea (54.2\%), anorexia (45.8\%), HFSR (43.8\%), weight loss (24.0\%), and hypertension (24.0\%). Dermatological AEs (HFSR and skin rash) occurred within 30 days for cabozantinib start in all but 2 patients. 
Fig. 1. OS in the study population. OS, overall survival; CI, confidence interval.
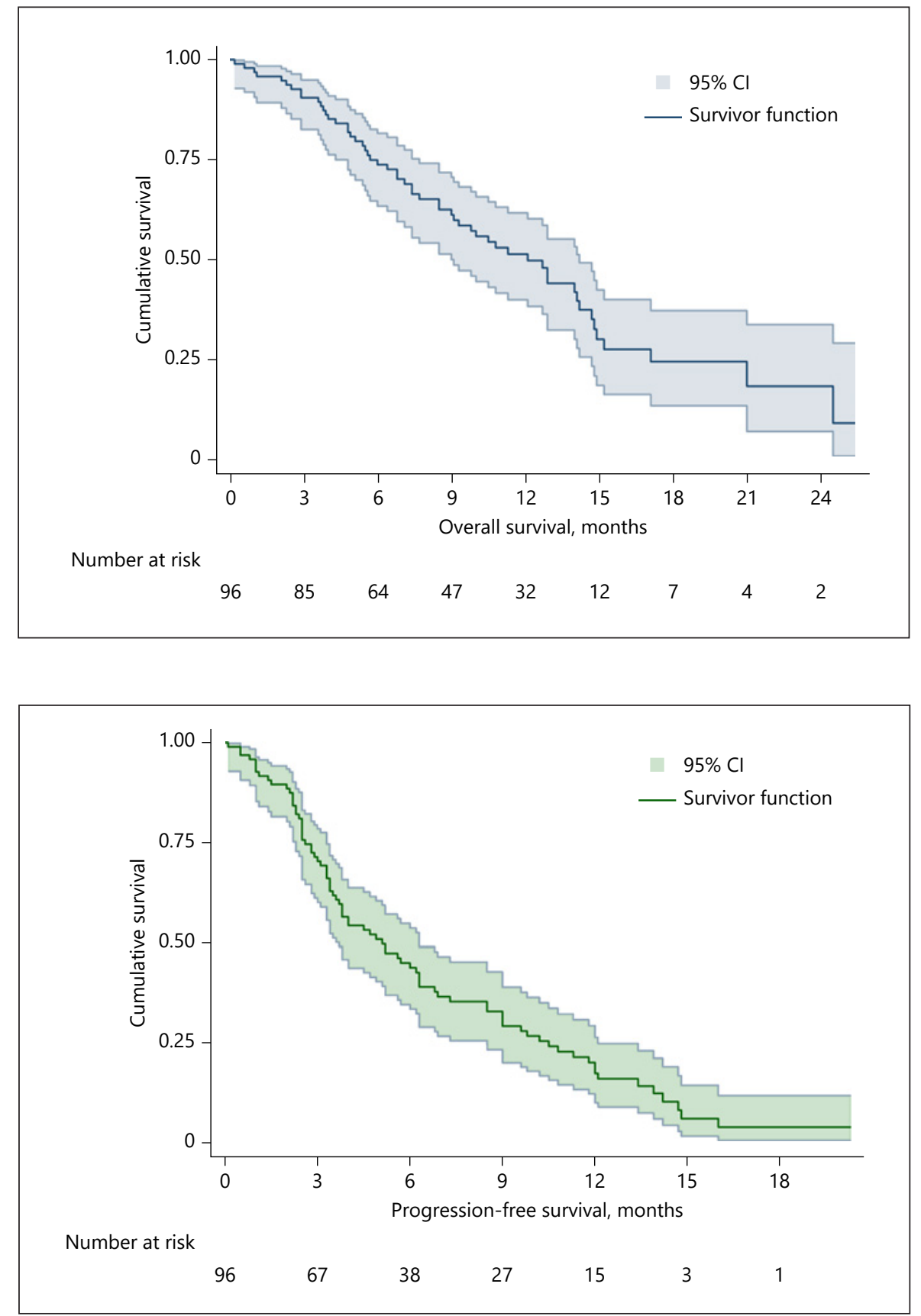

Fig. 2. PFS in the study population. PFS, progression-free survival; CI, confidence interval. cases of hepatic encephalopathy and the case of bullous erythrodysesthesia were attributed to cabozantinib. The rates of tRAEs of any grade, G3-G4 trAEs, and SAE were not different between patients receiving cabozantinib in the second and third-line setting.

\section{Dose Modifications}

Sixty-one patients $(63.5 \%)$ had to permanently reduce cabozantinib daily dose to $40 \mathrm{mg}$ daily due to AEs, with a 


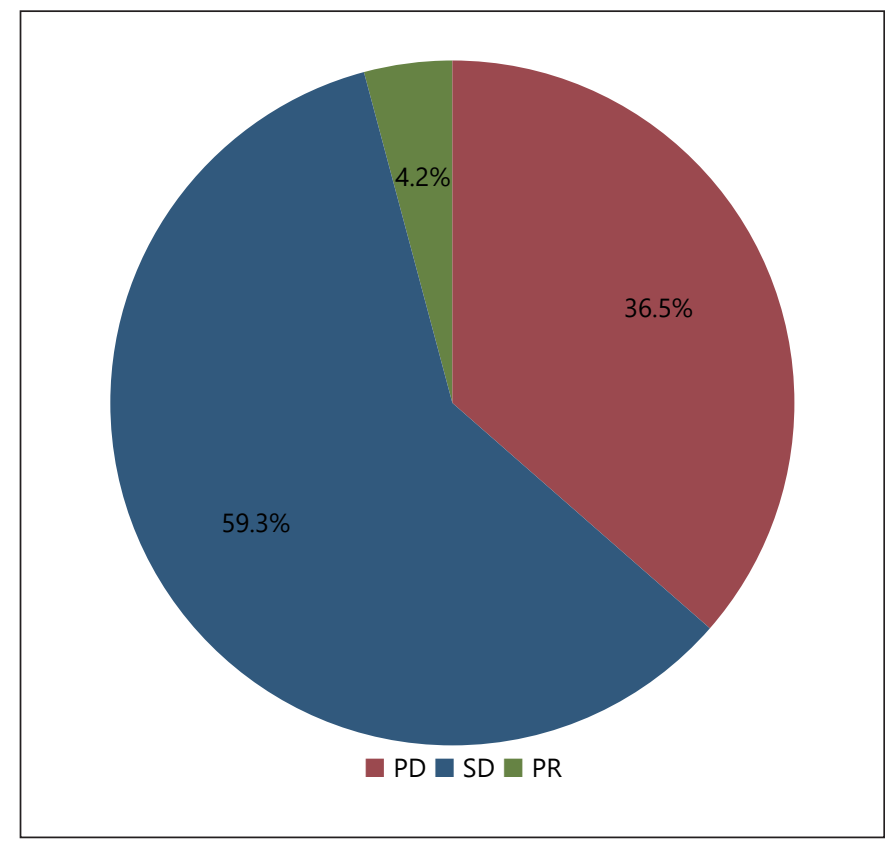

Fig. 3. Best radiological response according to the RECIST 1.1 in our study population. $\mathrm{PD}$, progressive disease; $\mathrm{SD}$, stable disease; PR, partial response; RECIST 1.1, Response Evaluation Criteria in Solid Tumours version 1.1.

median time to reduction of 43 days. Amongst these patients, a further dose reduction to $20 \mathrm{mg}$ daily was necessary for 19 cases (19.8\% of the total population).

Temporary drug interruptions were required in $66 \mathrm{pa}-$ tients $(68.8 \%)$, with a median duration of 13 days. The rate of permanent discontinuation due to unmanageable toxicities was $9.4 \%$. The median daily dose of cabozantinib was $49.6 \mathrm{mg}$ (IQR 29.0-60.0), for a median treatment duration of 5.1 months (IQR 2.7-9.6), accounting for a median cumulative dose of 7,056 mg (IQR 3,948$11,046)$. There were no differences in the treatment duration between patients receiving cabozantinib as a secondline or third-line drug $(p=0.248)$.

\section{Cabozantinib Permanent Discontinuation and \\ Subsequent Treatments}

A total of 77 patients (80.2\%) permanently discontinued cabozantinib at the end of this study. The reasons for discontinuation were progressive disease $(80.5 \%)$, intolerance (11.7\%), and liver decompensation (7.8\%).

Following the permanent discontinuation of cabozantinib, no patients received drugs approved for the treatment of HCC. However, a total of 7 patients (7.3\%) received off-label treatments, with metronomic capecitabine being the most common choice $(n=3)$. No patients received ICIs following cabozantinib discontinuation.

\section{Survival Correlates}

ECOG-PS >0, MVI, EHS, and AFP > $400 \mathrm{ng} / \mathrm{mL}$ were the baseline factors associated with an impaired survival at the univariate analysis (Table 3). The appearance of dermatological AEs, evaluated with a time-dependent analysis, was associated with a trend towards a higher OS, but without reaching a statistical significance (12.9 vs. 10.5 months, $p=0.143$ ). A separate analysis including only patients with early dermatological AEs (i.e., appeared within the first 30 days since cabozantinib start) and excluding the 2 cases of late dermatological AEs produced similar results $(p=0.135)$.

The multivariable Cox regression analysis confirmed ECOG-PS $>0, \mathrm{MVI}$, and AFP $>400 \mathrm{ng} / \mathrm{mL}$ as independent predictors of a shorter survival. Extrahepatic spread did not reach the statistical significance, probably due to the relatively low number of patients without metastasis and to some long-term responses amongst metastatic patients.

Patients who required a dose reduction for AEs (evaluated as time-dependent event) had a similar risk of progression (HR 1.061-95\% CI 0.661-0.703) and death (HR $0.788-95 \%$ CI $0.451-1.378$ ) compared to patients who remained on a full dose. The median OS stratified according to the radiological response was 14.8 (95\% CI 10.818.8 ) months for patients with disease control and 5.4 (95\% CI 4.2-668) months for patients with progressive disease $(p<0.001)$.

Amongst the 73 patients who permanently discontinued cabozantinib, the median PCS was 3.2 (95\% CI 2.53.9) months. The stratification according to the reason for discontinuation showed a median PCS of 7.8 (95\% CI 1.6-14.0) months for intolerance, 2.7 (95\% CI 1.9-3.5) months for progression, and 2.4 (95\% CI 1.2-3.6) months for liver failure $(p=0.070)$. Overall, patients who discontinued for intolerance had a better outcome in comparison with the other reasons for discontinuation (HR 0.493, 95\% CI 0.144-0.997, $p=0.048$ ).

Amongst the 74 patients who experienced a radiological progression, the median PPS was 4.2 (95\% CI 2.4-6.0) months. Patients with the BCLCp-B, BCLCp-C1, and BCLCp-C2 patterns of progression had a median PPS of 8.5 (95\% CI $0.0-18.6$ ), 8.5 (95\% CI $0.2-16.8$ ), and 3.4 (95\% CI 1.0-5.0) months, respectively. The difference between the BCLCp-C1 and BCLC-pC2 patterns was statistically significant $(p=0.020)$. With the strong limitations deriving from the post-hoc nature of the analysis and lim- 
Fig. 4. Overview of the therapeutic sequences of systemic drugs for HCC in our study population $(n=96)$.

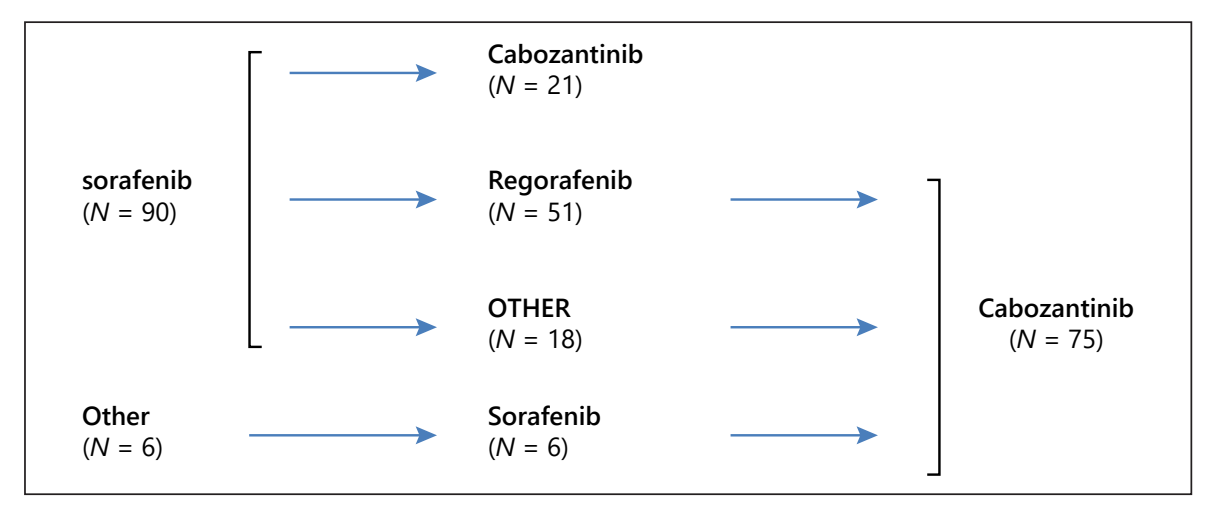

ited number of cases (allowing only univariate analyses), patients who had no extrahepatic spread/MVI $(p=0.034)$, ECOG-PS $=0(p=0.052)$, and AFP $<400 \mathrm{ng} / \mathrm{mL}(p=$ 0.023 ) at the start of sorafenib had a trend towards a better cumulative OS in the sorafenib-regorafenib-cabozantinib sequential treatment subgroup (see online suppl. Fig. 1; see www.karger.com/doi/10.1159/000515551 for all online suppl. material).

\section{Discussion}

The results of most registration trials of oncology drugs are often under scrutiny as their reproducibility in a real-life population is uncertain. Moreover, data about prognostic factors can be under-evaluated as the main focus of the trial remains the superiority against the competitor.

In this study, we provided real-life data of cabozantinib for HCC, to the best of our knowledge, for the first time after its approval for this medical condition. Moreover, we also provided fresh data about the main factors affecting the prognosis of HCC patients who received cabozantinib. Finally, we also explored the potential of sequential treatments of TKIs, which include cabozantinib in the second or third-line setting.

Baseline characteristics of our patients were similar to those of the CELESTIAL trial in terms of age, sex, ECOGPS, MVI, EHS, AFP $>400 \mathrm{ng} / \mathrm{mL}$, and duration of prior sorafenib treatment (online suppl. Table 1). Instead, most of our patients were treated in a third-line setting, in a striking difference with the CELESTIAL trial. As a consequence, a greater quote of our patients had received other systemic agents (in particular regorafenib) in comparison with the CELESTIAL trial [2].

First, we demonstrated that cabozantinib had efficacy and safety similar to those reported in the phase 3 CELES-
Table 2. Treatment emergent AEs which were interpreted as cabozantinib related amongst the study population $(n=96)$

\begin{tabular}{lll}
\hline AE & \multicolumn{2}{l}{ All grades (\%) Grade 3-4 (\%) } \\
\hline Fatigue & $65(67.7)$ & $6(6.3)$ \\
Diarrhoea & $52(54.2)$ & $3(3.1)$ \\
Hyporexia & $44(45.8)$ & $1(1.0)$ \\
Hand-foot skin reaction & $42(43.8)$ & $6(6.3)$ \\
Weight loss & $23(24.0)$ & $1(1.0)$ \\
Abdominal pain & $19(19.8)$ & $1(1.0)$ \\
Alanine aminotransferase increase & $19(19.8)$ & $6(6.3)$ \\
Mucositis & $17(17.7)$ & $2(2.1)$ \\
Dysphonia & $13(12.5)$ & $1(1.0)$ \\
Skin rash & $13(13.5)$ & $1(1.0)$ \\
Hypothyroidism & $11(11.5)$ & 0 \\
Nausea/vomiting & $9(9.4)$ & $1(1.0)$ \\
Thrombocytopaenia & $9(9.4)$ & $3(3.1)$ \\
Neutropaenia & $5(5.2)$ & $3(3.1)$ \\
Hepatic encephalopathy & $4(4.2)$ & $3(3.1)$ \\
Dysgeusia & $4(4.2)$ & 0 \\
Proteinuria & $4(4.2)$ & $1(1.0)$ \\
Fever & $3(3.1)$ & 0 \\
Anaemia & $2(2.0)$ & 0 \\
Venous skin ulceration & $1(1.0)$ & 0 \\
Amylase increase & $1(1.0)$ & $1(1.0)$ \\
Hyperthyroidism & $1(1.0)$ & 0 \\
\hline
\end{tabular}

Data are expressed as frequencies, \%. AE, adverse event.

TIAL trial. Differently from the registration trial, in which only $27 \%$ of the enrolled patients had received 2 previous treatments [2], the overwhelming majority of our patients received cabozantinib as a third-line agent. This difference was expected since patients in need for a second-line treatment and who were eligible for regorafenib could not receive cabozantinib in the NPU program. Of note, cabozantinib will likely be prescribed as a third rather than a second-line agent in the clinical practice in the next future 
Table 3. Predictors of survival according to the Cox regression

\begin{tabular}{llllll}
\hline HR & $\begin{array}{l}\text { Univariable } \\
95 \% \text { CI }\end{array}$ & $p$ value & Variable & HR & $\begin{array}{l}\text { Multivariable } \\
95 \% \text { CI }\end{array}$ \\
\hline 0.859 & $0.495-1.490$ & 0.588 & Age $>65$ years & - & - \\
1.161 & $0.598-2.256$ & 0.659 & Male sex & - & - \\
1.150 & $0.659-2.006$ & 0.658 & Sorafenib exposure $<6$ months & - & - \\
2.343 & $1.348-4.075$ & 0.003 & ECOG-PS $>0$ & 3.452 & $1.838-6.485$ \\
1.885 & $1.068-3.325$ & 0.026 & MVI & 1.803 & $1.022-3.183$ \\
1.733 & $0.927-3.239$ & 0.085 & Extrahepatic spread & 1.532 & $0.803-2.922$ \\
1.865 & $1.088-3.196$ & 0.023 & AFP $>400$ ng/mL & 2.377 & 0.001 \\
1.442 & $0.857-2.429$ & 0.168 & HBV aetiology & - & $-1.306-4.326$ \\
0.664 & $0.384-1.270$ & 0.143 & Dermatological AEs* & - & - \\
\hline
\end{tabular}

HR, hazard ratio; CI, confidence interval; ECOG-PS, Eastern Cooperative Group-Performance Status; AFP, alpha-fetoprotein; HBV, hepatitis B virus; MVI, macrovascular invasion; AE, adverse event. *Considered as a time-dependent variable.

(after atezolizumab-bevacizumab and sorafenib). Patients with contraindications to ICIs will also likely receive cabozantinib as a third rather than a second-line agent as it is approved for both settings, differently from regorafenib which can be prescribed in the second-line setting only. As expected in a third-line setting, our population consisted of difficult-to-treat patients, amongst whom the prevalence of EHS, MVI, and AFP $>400 \mathrm{ng} / \mathrm{mL}$ was high.

Nonetheless, a majority of patients achieved a disease control even in this challenging setting, with a radiological response (PR: 4 vs. $4 \%$, SD: 59 vs. $60 \%$, PD: 37 vs. $36 \%$ ) and a PFS (5.1 vs. 5.2 months) which were remarkably similar to those reported in the CELESTIAL trial [2]. The median OS of 12.1 months in our real-life population was slightly higher than the 10.2 months reported in the registration study. Differences of the same sing and magnitude had been reported for sorafenib, with an OS increasing from the 10.7 months reported in the registration trial $[5,15]$ to $>13$ months in subsequent real-life studies $[9$, 10] and phase 3 clinical trials [16]. With particular regard to our study, most patients were enrolled in centres which participated in the phase 3 trial of cabozantinib and had previous experience with the management of cabozantinib-related toxicities [17]. The tailored management of cabozantinib might be at least partially responsible for the small advantageous difference in the OS, as it has been shown for sorafenib $[18,19]$. This hypothesis is supported by the slightly higher duration of cabozantinib treatment (5.1 vs. 3.8 months), similar rate of dose reductions (64 vs. 62\%), and lower rate of patients permanently stopping cabozantinib for unmanageable AEs ( 9 vs. 16\%) in comparison with the CELESTIAL trial [2]. On the con- trary, post-cabozantinib treatments were prescribed in a very limited number of patients and are unlikely to have affected the OS. In this regard, our study confirmed that cabozantinib has an acceptable safety profile, similar to that reported for other TKIs $[9,10,15,16,20]$. Most toxicities were easily manageable with dose reductions and/ or temporary interruptions, while permanent discontinuation occurred in $<10 \%$ of cases. Also, no new safety signals were noted. The confirmation of an acceptable safety profile of cabozantinib is of particular interest as this agent is being tested in combination with different ICIs $[6,7]$, a scenario in which the risk of toxicities is increased.

Secondly, we reported prognostic factors associated with cabozantinib in a real-life setting. MVI, AFP $>400$ $\mathrm{ng} / \mathrm{mL}$, and ECOG-PS $>0$ were independently associated with impaired survival. However, a trend towards shorter survival was also observed for patients with EHS. The failure to confirm EHS and dermatological AEs as prognostics factors in the multivariable analysis probably depended on the relatively small sample of our study. Also, we found that discontinuation of cabozantinib for intolerance was associated with a better post-treatment prognosis, whereas a progression pattern characterized by the appearance of new extrahepatic lesions led to a worse post-progression outcome, similarly to sorafenib $[12,13]$. Taken together, all of these findings suggest that the prognostic factors for HCC patients receiving cabozantinib are similar to that of patients who are treated with sorafenib. The identification of prognostic and predictive factors is of critical importance to improve the selection of patients and to correctly inform them about the risk/ benefit ratio, both in terms of life expectancy and quality 
of life. The CELESTIAL trial showed a small reduction in quality-adjusted life years in the cabozantinib treatment arm in comparison with placebo at Day 50 after the randomization [21]. This pattern reversed at Day 100 and Day 150, with an overall advantage of cabozantinib versus placebo in terms of quality-adjusted life years [21]. Indeed, the optimal management of these patients should rely on predictive rather than prognostic information. In this way, there would be the possibility to identify patients for whom the treatment is futile on 1 hand (also reducing the problem of cost-effectiveness of cabozantinib) [22, 23] and patients with the potential of becoming long survivors on the other hand. Unfortunately, such biomarkers have to be identified yet in the case of TKIs for HCC and in particular of cabozantinib.

Despite the similarities with other TKIs in terms of safety and prognostic factors, cabozantinib maintained a promising antineoplastic activity also in TKI-experienced patients in our study. The median cumulative survival of the sorafenib-regorafenib-cabozantinib sequence was about 36 months, representing a further extension of life expectancy in comparison with the reported cumulative OS of 26 months of the sorafenib-regorafenib sequence [14]. Indeed, only a minority of patients who start a frontline systemic treatment will still be eligible for further treatment following the failure of 1 or 2 previous lines. For instance, Fung et al. [24] reported that only $13.1 \%$ of 720 sorafenib-experienced patients were eligible for cabozantinib, regorafenib, or ramucirumab as a second-line treatment if strict eligibility criteria were used. If the eligibility criteria were extended to also include patients with ECOG-PS 2 or Child-Pugh class B7, then the eligibility rate increased to $31 \%$, but with a lesser survival expectancy [24]. The proportion of patients theoretically eligible for a third-line treatment has never explicitly been investigated, but the proportion is expected to be even lower than that reported for the second-line setting. Since the deteriorating liver function was the main reason precluding the eligibility of patients for second-line treatments in Fung et al.'s [24] study, the opportunity to avoid reiterated locoregional treatments in non-responding patients should be further stressed.

Our study has some limitations deserving discussion. Firstly, the relatively limited number of patients and length of follow-up might have prevented the identification of further prognosticators. Second, our findings cannot be extended to the patients who deteriorated their liver function during the previous lines of treatment (with particular regard to Child-Pugh B patients) as only patients with preserved liver function were included, simi- larly to the CELESTIAL trial. Caution should be also used in the interpretation of the cumulative survival of patients who received 2 previous lines of treatment, due to the relatively small sample size for each sequence and to the possibility that patients reaching a third-line setting still in optimal conditions might theoretically have a relatively benign tumour biology (even though this hypothesis is difficult to prove or disprove without preclinical and translational evidence).

In conclusion, we found that cabozantinib maintained the efficacy and safety profile reported in its registration trial also in a difficult-to-treat real-life Western population, mainly in a third-line scenario. More importantly, we showed that cabozantinib share most of the prognostic factors with other TKIs used for the treatment of HCC and that the sequential treatment sorafenib-regorafenibcabozantinib can lead to a median life expectancy of about 3 years. While the current therapeutic scenario of HCC will change in the next future with the advent of the atezolizumab-bevacizumab combination, our data can have a long-term relevance as cabozantinib is being tested in the frontline setting and, in any case, remains a viable third-line option in case of failure of both atezolizumabbevacizumab and subsequent sorafenib.

\section{Statement of Ethics}

The study protocol was reviewed and approved by the local Ethics Committee (EC). In particular, the protocol was firstly approved by the Bologna Authority Hospital EC (protocol 202.2020. Oss.AOUBo) for the coordinating centre and subsequently approved by the remaining EC. A written informed consent was obtained according to the EC's recommendations. The study was conducted according to the ethical guidelines of the 1975 Declaration of Helsinki.

\section{Conflict of Interest Statement}

F.To.: consultant for Bayer; advisory board for Guerbet. V.D.: personal fees from Bayer, MSD, Eisai, and Ipsen. L.R.: consulting fees from Amgen, ArQule, AstraZeneca, Basilea, Bayer, Celgene, Eisai, Exelixis, Hengrui, Incyte, Ipsen, Lilly, MSD, Nerviano Medical Sciences, Roche, Sanofi; lectures fees from AbbVie, Amgen, Eisai, Gilead, Incyte, Ipsen, Lilly, Roche, Sanofi; travel fees from Ipsen; and institutional research funding from Agios, ARMO BioSciences, AstraZeneca, BeiGene, Eisai, Exelixis, Fibrogen, Incyte, Ipsen, Lilly, MSD, and Roche. M.I.: speaking and teaching for Bayer, Gilead Science, Janssen, BTG, AbbVie; consultant for BCG; Editorial Board Member of Liver Cancer. F.M.: consultant for Bayer and Ipsen, travel grants from Bayer. B.D.: honoraria from Ipsen, AstraZeneca, Incyte, Lilly, Eisai, Bayer, Roche, and MSD. F.Tr.: advisory board for Bayer, Alfasigma, Bristol-Myers Squibb, and 
Sirtex. G.C.: advisory board for Bayer; V.Z.: advisory board for Bristol-Myers Squibb and Merck; speakers' bureau for AstraZeneca and Lilly; personal fees from Bayer, Roche, and Servier. G.B.: advisory board for Eli Lilly and MSD. T.P. institutional research funding from Eli Lilly. F.P.: consultant for Astrazeneca, Bayer AG, EISAI, GE, and Tiziana life sciences; speaker's bureau honoraria from Bayer AG, Bracco, EISAI, and Laforce; and research contract with Esaote; Massimo Iavarone and Fabio Piscaglia are Editorial Board Members of Liver Cancer. The remaining authors declared no conflict of interests.

\section{Funding Sources}

The authors did not receive any funding.

\section{Author Contributions}

Design and concept: F.To. and F.P.; data curation: all authors; data collection: F.To., V.D., S.D., I.G., F.Tr., C.M., F.D., M.P.B., S.P., G.C., A.G., M.D.R., T.P., P.F., C.V., I.B., and C.C.; statistics: F.To. and A.G.; drafting the manuscript: F.To.; critical revision: V.D., L.R., F.P., S.D., M.I., I.G., C.M., F.D., and G.C. All authors approved the final version of the manuscript.

\section{References}

1 Yakes FM, Chen J, Tan J, Yamaguchi K, Shi Y, $\mathrm{Yu}$ P, et al. Cabozantinib (XL184), a novel MET and VEGFR2 inhibitor, simultaneously suppresses metastasis, angiogenesis, and tumor growth. Mol Cancer Ther. 2011 Dec; 10(12):2298-308.

2 Abou-Alfa GK, Meyer T, Cheng AL, ElKhoueiry AB, Rimassa L, Ryoo BY, et al. Cabozantinib in patients with advanced and progressing hepatocellular carcinoma. $N$ Engl J Med. 2018 Jul;379(1):54-63.

3 Bouattour M, Mehta N, He AR, Cohen EI, Nault JC. Systemic treatment for advanced hepatocellular carcinoma. Liver Cancer. 2019;8(5):341-58.

4 Rimassa L, Pressiani T, Merle P. Systemic treatment options in hepatocellular carcinoma. Liver Cancer. 2019 Nov;8(6):427-46.

5 Finn RS, Qin S, Ikeda M, Galle PR, Ducreux M, Kim TY, et al. Atezolizumab plus bevacizumab in unresectable hepatocellular carcinoma. N Engl J Med. 2020 May 14;382(20): 1894-905.

6 Kelley RK, W Oliver J, Hazra S, Benzaghou F, Yau T, Cheng AL, et al. Cabozantinib in combination with atezolizumab versus sorafenib in treatment-naive advanced hepatocellular carcinoma: COSMIC-312 Phase III study design. Future Oncol. 2020 Jul;16(21):1525-36.

7 Yau T, et al. Nivolumab (NIVO) + ipilimum$\mathrm{ab}$ (IPI) + cabozantinib (CABO) combination therapy in patients (pts) with advanced hepatocellular carcinoma (aHCC): results from CheckMate 040. J Clin Oncol. 2020;38(4):478.

8 Iavarone M, Cabibbo G, Piscaglia F, Zavaglia C, Grieco A, Villa E, et al. Field-practice study of sorafenib therapy for hepatocellular carcinoma: a prospective multicenter study in Italy. Hepatology. 2011 Dec;54(6):2055-63.

9 Marrero JA, Kudo M, Venook AP, Ye SL, Bronowicki JP, Chen XP, et al. Observational registry of sorafenib use in clinical practice across Child-Pugh subgroups: the GIDEON study. J Hepatol. 2016;65(6):1140-7.
10 Ganten TM, Stauber RE, Schott E, Malfertheiner P, Buder R, Galle PR, et al. Sorafenib in patients with hepatocellular carcinoma-results of the observational INSIGHT Study. Clin Cancer Res. 2017 Oct;23(19):5720-8.

11 Eisenhauer EA, Therasse P, Bogaerts J, Schwartz LH, Sargent D, Ford R, et al. New response evaluation criteria in solid tumours: revised RECIST guideline (version 1.1). Eur J Cancer. 2009 Jan;45(2):228-47.

12 Reig M, Rimola J, Torres F, Darnell A, Rodriguez-Lope C, Forner A, et al. Postprogression survival of patients with advanced hepatocellular carcinoma: rationale for second-line trial design. Hepatology. 2013 Dec;58(6):2023-31.

13 Iavarone M, Cabibbo G, Biolato M, Della Corte C, Maida M, Barbara M, et al. Predictors of survival in patients with advanced hepatocellular carcinoma who permanently discontinued sorafenib. Hepatology. 2015 Sep; 62(3):784-91.

14 Finn RS, Merle P, Granito A, Huang YH, Bodoky G, Pracht M, et al. Outcomes of sequential treatment with sorafenib followed by regorafenib for HCC: additional analyses from the phase III RESORCE trial. J Hepatol. 2018;69(2):353-8.

15 Llovet JM, Ricci S, Mazzaferro V, Hilgard P, Gane E, Blanc JF, et al. Sorafenib in advanced hepatocellular carcinoma. N Engl J Med. 2008 Jul;359(4):378-90.

16 Kudo M, Finn RS, Qin S, Han KH, Ikeda K, Piscaglia F, et al. Lenvatinib versus sorafenib in first-line treatment of patients with unresectable hepatocellular carcinoma: a randomised phase 3 non-inferiority trial. Lancet. 2018 Mar 24;391(10126):1163-73.

17 Rimassa L, Danesi R, Pressiani T, Merle P. Management of adverse events associated with tyrosine kinase inhibitors: improving outcomes for patients with hepatocellular carcinoma. Cancer Treat Rev. 2019 Jul;77:20-8.

18 Tovoli F, Ielasi L, Casadei-Gardini A, Granito A, Foschi FG, Rovesti G, et al. Management of adverse events with tailored sorafenib dosing prolongs survival of hepatocellular carcinoma patients. J Hepatol. 2019;71(6):1175-83.
19 Raoul JL, Adhoute X, Penaranda G, Perrier H Castellani P, Oules V, et al. Sorafenib: experience and better manage-ment of side effects improve overall survival in hepatocellular carcinoma patients: a real-life retrospective analysis. Liver Cancer. 2019 Nov;8(6):457-67.

20 Bruix J, Qin S, Merle P, Granito A, Huang YH, Bodoky G, et al. Regorafenib for patients with hepatocellular carcinoma who progressed on sorafenib treatment (RESORCE): a randomised, double-blind, placebo-controlled, phase 3 trial. Lancet. 2017 Jan 7;389(10064):56-66.

21 Abou-Alfa GK, Mollon P; Memorial Sloan Kettering Cancer Center NY; Ipsen A; University College London Cancer Institute L, National Taiwan University Hospital and $\mathrm{Na}$ tional Taiwan University Cancer Center T, University of Southern California Norris Comprehensive Cancer Center LA, et al. Quality-adjusted life years assessment using cabozantinib for patients with advanced hepatocellular carcinoma (aHCC) in the CELESTIAL trial. [cited 2020 Aug 19]. Available from: https://meetinglibrary.asco.org/record/ 168580/abstract.

22 Shlomai A, Leshno M, Goldstein DA. Cabozantinib for patients with advanced hepatocellular carcinoma: a cost-effectiveness analysis. Therap Adv Gastroenterol. 2019;12: 175628481987830.

23 Soto-Perez-de-Celis E, Aguiar PN, Cordón ML, Chavarri-Guerra Y, de Lopes GL. Costeffectiveness of cabozantinib in the secondline treatment of advanced hepatocellular carcinoma. J Natl Compr Cancer Netw. 2019 Jun 1;17(6):669-75.

24 Fung AS, Tam VC, Meyers DE, Sim HW, Knox JJ, Zaborska V, et al. Second-line treatment of hepatocellular carcinoma after sorafenib: characterizing treatments used over the past 10 years and real-world eligibility for cabozantinib, regorafenib, and ramucirumab. Cancer Med. 2020 Jul;9(13):4640-7. 